\title{
Relationship between Interpersonal Relationship Distress and Pet Attachment: Chain mediating Effects of Coping Style and Loneliness among the Undergraduates
}

\author{
Hou Yongmei \\ Staff Room of Psychology, \\ School of Humanities and Management, \\ Guangdong Medical University, \\ Dongguan 523808, Guangdong Province, China \\ 352314640@qq.com
}

\author{
Hu Peicheng \\ Department of Psychology \\ Health Science Center of Peking University \\ Beijing 100083, China
}

\author{
Huang Wanhui \\ Staff Room of Psychology, \\ School of Humanities and Management, \\ Guangdong Medical University, \\ Dongguan 523808, Guangdong Province, China
}

\begin{abstract}
To explore the relationship among interpersonal relationship, coping style tendency, loneliness and pet attachment among the undergraduates. College Students Interpersonal Comprehensive Diagnostic scale (CSICDS), Simplified Coping Style Questionnaire (SCSQ), UCLA Loneliness Scale (UCLALS) and Lexington Attachment to Pets Scale (LAPS) were adminis tered to 547 undergraduate pet owners who were selected by stratified random sampling from 7 universities in Guangzhou City. (1) The total score of CSICDS positively predict the total score of LAPS $(\mathrm{r}=\mathbf{0 . 1 6 0}, \mathbf{P}<\mathbf{0 . 0 1})$. (2) The coping style tendency score had mediating effect between the total score of CSICDS and UCLALS, while the total score of UCLALS had mediating effect in the relationship between coping style tendency score and the total score of LAPS. (3) The total score of CSICDS had an indirect effect on the undergraduates' pet attachment through the coping style tendency-loneliness mediating chain. Interpersonal relationship distress not only had a direct role on undergraduates' pet attachment, but also indirectly influenced it through the coping style tendency-loneliness mediating chain.
\end{abstract}

Keywords-Interpersonal Relationship Distress; Pet attachment; loneliness; Coping style; Undergraduates

In recent years, more and more college students have kept pets. Although colleges and universities have enacted prohibition orders to forbid students keeping pets or small animals in the dormitory, some college students still persist their old ways. It is known that more than $12 \%$ of college students keep pets in the dormitory, which has a non-negligible impact on the maintenance of the environment and the lives of them and roommates ${ }^{[1-2]}$. It can be said that keeping pets is one of the important conduct problems of college students.
There is no consistent idea about the role of pets. However, many scholars believe that pets can provide special, multi-faceted attachment to the keeper, that is, pet attachment, which is similar to parental attachment and intimate attachment that are beneficial to physical and mental health at all stages of life ${ }^{[3-6]}$. Specifically, people who have pet attachment are often confused by interpersonal problems and loneliness ${ }^{[7]}$. Through the benign interaction with the keeper, the pet can provide non-humanized social support for the keeper ${ }^{[3-4]}$, make up for the interpersonal relationship, thereby relieving the psychological stress of the keeper, alleviating the bad feelings such as loneliness ${ }^{[8-10]}$, and improving their physical and mental health ${ }^{[11-12]}$.

Loneliness appears when one perceives his social contact is not as good as his own expectation, accompanied by the pains of one's unaccepted by others which is caused by isolation or lack of contact with others ${ }^{[13]}$. Loneliness is an important predictor of mental health and behavioral problems ${ }^{[14-16]}$, and is also an element for psychosomatic diseases ${ }^{[17-18]}$. In the life, the influence of loneliness on the individual is like an " $\cap$ ", reaching a peak in adolescence ${ }^{[19]}$. College students in adolescence generally have a high level of loneliness. Almost everyone in China has loneliness, and the incidence of moderate or severe loneliness is 61.4 to $95.5 \%{ }^{[20-22]}$.

Interpersonal relationship is a direct and sensible emotional connection between people through interaction and exchanges. It reflects the needs of individual affiliation ${ }^{[23]}$. Interpersonal relationship embodies individual's social resource and is an important component of the social support system. However, interpersonal relationship is the major problem faced by college students, as $38.6 \%$ of college students have 
interpersonal relationship problems, and $9.1 \%$ of college students have serious ones ${ }^{[24]}$. Poor interpersonal adaptability, lack of coping capacity and social skills, and poor interpersonal relationships are the main causes of loneliness ${ }^{[25-26]}$.

Response is the mediator of stress and mental health, and also the mediator of mentality and emotional and behavioral problems with a buffering effect. A skillful response helps solve problems, relieve mental stress, and plays an important role in protecting the body and mind. While a bad response impairs the individual's physical and mental health ${ }^{[27]}$.

Previous studies have found that there is a significant correlation between coping styles on the one hand, and pet attachment, interpersonal relationships, and loneliness on the other hand. The coping style of problem-solving is significantly positively correlated with pet attachment [28] Interpersonal relationship puzzle is the mediator between coping styles and college students' depression ${ }^{[29]}$. Both mature and immature coping styles play a partial mediating role in loneliness and self-harmony, but in opposite direction ${ }^{\text {[30] }}$. Poor interpersonal adaptability, lack of coping skills and social skills, and poor interpersonal relationships are the main causes of loneliness ${ }^{[25-26]}$. And pet attachment is negatively correlated with loneliness ${ }^{[8-10]}$. What's more, pet attachment can promote interpersonal interactions among pet keepers and improve their interpersonal relationships ${ }^{[31]}$.

In summary, the cause of pet attachment is loneliness, and interpersonal relationship is an important cause of loneliness, and coping style is a mediator of many mental health problems (including loneliness and interpersonal relationship). Moreover, pet attachment is an explicit behavior of the individual and is the outcome variable. Interpersonal relationship is the deep-rooted and distal mentality (anterior dependent variable), while loneliness and coping style are superficial and proximal mentality. Interpersonal relationship is more mediated by the coping style and loneliness. In this way, we can assume that coping style and loneliness play a chain intermediary role between interpersonal relationship puzzles and pet attachment.

\section{OBJECTS AND TOOLS}

\section{A. Objects}

From April to May 2018, stratified random sampling method was used to select 600 undergraduates who kept pets by themselves or at home. These students are from Guangdong Pharmaceutical University, Jinan University, Guangzhou Academy of Fine Arts, Guangdong University of Technology, Xinghai Conservatory of Music, Guangdong Sport University and Guangdong University of Foreign Studies. 547 valid questionnaires were collected with $91.2 \%$ effective questionnaire. There are 247 male students and 300 female students. 173 of them are the only child in their own home. 342 are from urban and 205 from rural areas. 163 are freshmen, 141 seniors, 120 juniors, and 123 seniors. The age ranges from 17 to 24 , with an average of $(19.82 \pm 1.51)$.

\section{B. Tools}

\section{1) Lexington Attachment to Pets Scale (LAPS)}

LAPS, compiled by Lexington (1992) ${ }^{[3]}$ and translated to Chinese and revised by $\mathrm{Wu}$ Xiaomin ${ }^{[32]}$, is used to assess the intimacy between pet keepers and pets. The scale consists of 23 entries, of which entry 8 and 21 are scored in reverse which means that the higher the score, the greater the pet's attachment In the Likert 4 rating, " 1 " is "strongly disagree" and " 4 " is "totally agreed". The total mean scores of the scales " 1 ", " 2 ", "3", "4" represent "low pet attachment", "general pet attachment", "high pet attachment" and "extremely high pet attachment" respectively. In this study, the Cronbach'a coefficient of the scale was 0.854 , and the test-retest reliability after one month was 0.826 .

2) Revised UCLA Loneliness Scale-Version 3 (UCLALS)

UCLA Loneliness Scale was composed by Russell and others (1978), and translated and revised by Liu Ping ${ }^{[33]}$. It consists of 20 entries, including 11 "lonely" entries and 9 "non-lonely" reverse order entries. In the Likert 4 rating, " 1 " is "never (so)" and " 4 " is "always (so)". If the total score is higher than 44, then it is highly lonely. If the total score is lower than 28, it is low loneliness. And the score falls in the range from 28 to 44 , representing moderate loneliness ${ }^{[33]}$. In the study, the Cronbach'a coefficient of the scale was 0.914, and the test-retest reliability after one month was 0.875 .

\section{3) Simplified Coping Style Questionnaire (SCSQ)}

SCSQ was compiled by Xie Yaning (1990) ${ }^{[34]}$, and it was a self-assessment scale with a total of 20 entries that involve different attitudes and measures that may be taken in daily life. And it is divided into two subscales of positive response and negative response. Using the Likert 4 rating, it is divided into four categories: "not used", "occasionally used", "sometimes used" and "usually used" (scoring 0, 1, 2, 3 respectively). which will actively respond to the subscale. The standard score of active response minus that of the negative response is the coping style tendency score (the positive score indicates that the individual's coping style is positive while the negative number indicates negative coping style). In this study, the Cronbach'a coefficient of the total scale is 0.881 ; the Cronbach'a coefficient of the positive response subscale is 0.868; the Cronbach'a coefficient of the negative response subscale is 0.785 . The test-retest reliability of the total scale one month later is 0.850 , while the positive response subscale and the negative response subscale are 0.832 and 0.820 respectively.

\section{4) College Students Interpersonal Comprehensive} Diagnostic Scale (CSICDS)

CSICDS was developed by Zheng Richang et al. (1999) [35] to measure the degree of interpersonal relationships puzzles and related behavioral distress. A total of 28 questions are mainly in four dimensions: talking with people, making friends, dealing with people and heterosexual interactions. Adopt the "yes-no" scoring system in which "yes" gets one point while "no" means no point. The higher the score, the more serious the one puzzled by interpersonal relationship. According to the total score, it can be divided into three levels: few or no communication trouble (0-8 points), a certain degree of communication distress (9-14 points), and serious communication problems (15-28 points). In the study, the Cronbach'a coefficient of the scale was 0.847, and the Cronbach'a coefficient of each subscale was 0.791 0.821. The test-retest reliability of the total scale one month later was 
0.853 , and the test-retest reliability of the four subscales was 0.802 to 0.839 .

\section{Data Processing}

Adopt SPSS 20.0 to analyze the valid data. Descriptive statistics were used to calculate the average score and standard deviation of the subjects on each scale. Pearson Product Moment Correlation Coefficient was used to explore the correlation between variables. AMOS22.0 was used to construct the structural equation modeling. And the median effect test was performed by the Bootstrap method.

\section{RESUlts}

\section{A. College Students'Scores and Correlation Analysis on Four Scales}

It can be seen from Table 1 that the pet attachment level of this group of college students is low, and the coping style tends to be intermediate. They have a certain degree of (obvious) communication trouble, and high degree of loneliness.

From Table 1, we know that the total score of loneliness is significantly positively correlated with the total score of the pet attachment scale and the interpersonal replacement factor. The total score of pet attachment is significantly negatively correlated with coping style scale (coping style tendency) and positive response subscale scores, and is positively correlated with negative response subscale scores, as well as the total scores of college students' interpersonal relationship scale and the scores of the friends-making, dealing with people, heterosexual interaction subscales respectively. There is a significant positive correlation between the total score of the loneliness scale and the scores of the comprehensive scale of the interpersonal relationship and the scores of the four factors. What's more, the total score of the loneliness scale has a positive correlation with the total score of coping style scale (the coping tendency) and the positive response subscale score, and a negative correlation with the negative response subscale score. The average score of the positive response subscales is negatively correlated with the total score of the interpersonal relationship scales and the scores of each dimension, while the average scores of the negative response scale is significantly positively correlated with these scores.

TABLE I. DESCRIPTIVE ST ATISTICS AND CORRELATION ANALYSIS OF RESEARCH VARIABLES

\begin{tabular}{|c|c|c|c|c|c|c|c|c|c|c|c|c|c|c|c|}
\hline & & $\mathrm{M}$ & SD & 1 & 2 & 3 & 4 & 5 & 6 & 7 & 8 & 9 & 10 & 11 & 12 \\
\hline 1 & Positive response & 1.95 & .46 & & & & & & & & & & & & \\
\hline 2 & Negative response & 1.30 & .54 & $-.238 * *$ & & & & & & & & & & & \\
\hline 3 & Response inclination & -.022 & 1.50 & $.788 * *$ & $-.786 * *$ & & & & & & & & & & \\
\hline 4 & Total score of loneliness & 44.51 & 7.87 & $-.572 * *$ & $.684 * *$ & $-.799 * *$ & & & & & & & & & \\
\hline 5 & Conversation & 2.53 & 1.81 & $-.395 * *$ & $.349 * *$ & $-.473 * *$ & $.576 * *$ & & & & & & & & \\
\hline 6 & Contact & 3.32 & 2.05 & $-.235 * *$ & $.379 * *$ & $-.390 * *$ & $.500 * *$ & $.628 * *$ & & & & & & & \\
\hline 7 & Dealing with people & 1.51 & 1.44 & $-.295 * *$ & $.319 * *$ & $-.390 * *$ & $.432 * *$ & $.479 * *$ & $.478 * *$ & & & & & & \\
\hline 8 & Opposite-sex interaction & 1.77 & 1.69 & $-.270 * *$ & $.323 * *$ & $-.376 * *$ & $.398 * *$ & $.531^{* *}$ & $.565 * *$ & $.426 * *$ & & & & & \\
\hline 9 & Interpersonal communication & 9.12 & 5.62 & $-.369 * *$ & $.429 * *$ & $-.508 * *$ & $.598 * *$ & $.833^{* *}$ & $.859 * *$ & $.712 * *$ & $.786 * *$ & & & & \\
\hline 10 & General attachment & 20.71 & 5.61 & -.049 & .045 & -.060 & .059 & .033 & $.141^{* *}$ & .075 & .072 & $.103^{*}$ & & & \\
\hline 11 & Interpersonal substitute & 10.43 & 3.38 & $-.193 * *$ & $.222 * *$ & $-.264 * *$ & $.224 * *$ & $.108^{*}$ & $.131 * *$ & $.143 * *$ & $.114 * *$ & $.153^{* *}$ & $.650 * *$ & & \\
\hline 12 & Pet status & 10.56 & 2.43 & -.007 & $.108^{*}$ & -.073 & .047 & .017 & $.146^{* *}$ & .063 & .020 & $.081^{*}$ & $.733 * *$ & $.472^{* *}$ & \\
\hline 13 & Total score of attachment & 41.71 & 10.05 & $-.094 *$ & $.126^{* *}$ & $-.140 * *$ & $.120 * *$ & .058 & $.158^{* *}$ & $.105^{*}$ & $.083 *$ & $.160^{* *}$ & $.953 * *$ & $.813^{* *}$ & $.809 * *$ \\
\hline
\end{tabular}

Note: ${ }^{*} \mathrm{p}<0.05, * * \mathrm{p}<0.01, * * * \mathrm{p}<0.001$ (the same below)

\section{B. The Verification on the Mediation Effect of Response and Sense of Loneliness in College Students' Interpersonal relationships and Pet Attachment}

With a comprehensive scale of college students' interpersonal relationships being an independent variable, the reaction's propensity score and the total value of loneliness scale being the mediating variable as well as the total value of pet attachment scale being the dependent variable, the model fitted well under the usage of AMOS22.0 structural equation model $(\mathrm{df}=1, \chi 2=2.635$, the significance probability value $\mathrm{P}=0.105>0.05$, R MSEA $=0.055$, GFI $=0.998$, AGFI $=$ 0.976, NFI=0.997, IFI $=0.998, \mathrm{PGFI}=1.000$ ). From figure 1 , we find that the comprehensive scale of college students' interpersonal relationships can not only directly predict the total value of the pet attachment scale, but also calculate it through the mediation effect of loneliness and do it in an indirect way with the chain mediating effect of coping inclination and loneliness.

After controlling the factors of gender and whether being an only child, Bootstrap, a non-parametric percentile method for deviation correction, was used to verify the mediation effect, in which 200 samples were randomly selected from the original data and $95 \%$ credibility interval was calculated. As the result showed that $95 \%$ credibility interval of the reaction inclination and loneliness in the indirect effect between the total value of college students' interpersonal relationships scale and that of pet attachment scale was $[0.04,0.09]$, excluding 0 , the linkage mediating effect has statistics significance. Among the data, the indirect effect value of reaction inclination and loneliness in both total values of college students' inter personal relationships scale and pet attachment scale was 0.044, accounting for $18.6 \%$ of the total effect. 


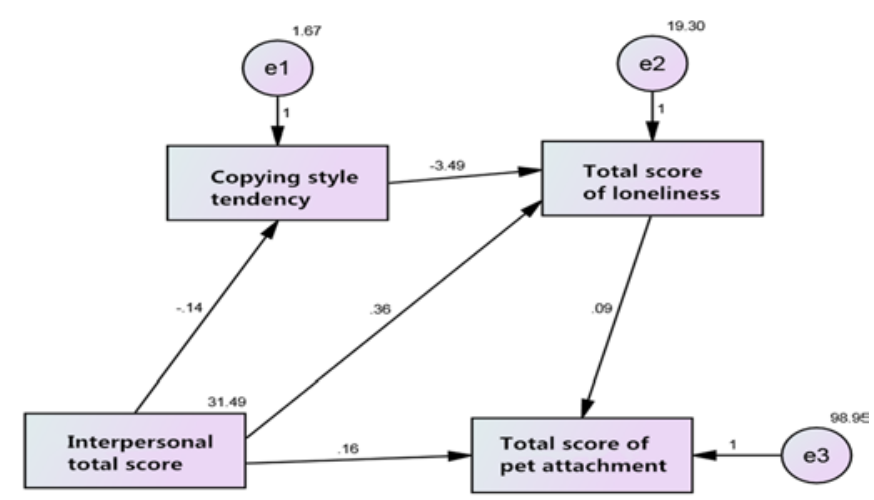

Fig. 1. The model of chain mediation between coping styles and loneliness in college students' interpersonal relationships and pet Attachment

\section{DISCUSSION}

This study found that the pet attachment level of college pet keepers is low, and the coping style tends to be intermediate. These students have a high degree of loneliness and some certain interpersonal relationship puzzles. It is suggested that these pet owners don't have deep sentiment on their pets. Meanwhile, they also have a coping style that is not active enough, obvious interpersonal relationship problems and serious loneliness.

This study also found that there are direct effect and an indirect effect between the interpersonal relationship problems and pet attachment.

On the one hand, there is a significant positive correlation between interpersonal relationship problems and pet attachment, which is the direct effect between the two, and is consistent with the results of previous studies ${ }^{[7]}$. In other words, students who have worse interpersonal relationship are more inclined to keep pets to seek psychological comfort and support. This is because pets can be regarded as a special social support to supplement the individual interpersonal support ${ }^{[3-4]}$.

On the other hand, there is an indirect effect between the perplexity of interpersonal relationships and pet attachment. Two ways demonstrate the effect: one is about interpersonal relationships perplexity — loneliness — pet attachment, where loneliness plays a mediating role; the other is about interpersonal relationships perplexity —reaction -loneliness - pet attachment, where both of reaction and loneliness take the chain mediating effect, representing that without positive and effective reaction, the interpersonal relationships sufferer arise a sense of loneliness and get worse to be pet-attaching. For the first way, we can comprehend it from a view of sentimental theory: due to interpersonal relationships perplexity, the individual feels insufficient in interpersonal support and therefore feel lonely directly, which can be verified from the definition of loneliness ${ }^{[13]}$ and results of other researches ${ }^{[25-26]}$. In order to release the sense of loneliness, the individual begins to be pet-attaching. As for the second way, we can stand on the view of reaction' s buffer mechanism: since interpersonal relationships perplexity is a negative life event, the key to decide whether it causes individual emotional problems (like sense of loneliness) and behavior problems (pet attachment) is mental buffer mechanism, especially whether an individual has a positive and effective reaction. If the individual does have it (such as working out reasonable attributions, solving the interpersonal issues in a positive way or distracting attention by learning or entertaining), they won't arise serious sense of loneliness and even become pet-attaching; on the contrary, if the individual fail to generate such effective reaction, then they will fall in strong loneliness and worsen to be pet-attaching.

\section{CONCLUSION}

This study reveals the relationship between college students' interpersonal relationship distress, coping style, loneliness and pet attachment, and verifies the theoretical hypothesis that coping style and loneliness play a chain intermediary role in interpersonal relationship distress and pet attachment. On the one hand, there is a significant positive correlation between interpersonal relationship and pet attachment, which is the direct effect between the two. On the other hand, there is an indirect effect between interpersonal relationship and pet attachment. It is manifested in the following two ways. The first is interpersonal relationship troubles-looseness-pet attachment and loneliness plays a mediating role in interpersonal relationship troubles and pet attachment. The second is interpersonal relationship troublescoping style - loneliness - pet attachment, and coping style and loneliness play the chain intermediary role between interpersonal relationship troubles and pet attachment.

To sum up, it can be seen that the real reason for college students to raise pets is to reduce the loneliness and anxiety caused by their inability to cope with interpersonal relationships. Given this, we put forward the following reference opinions for family and school education. To avoid students keeping pets, we should start from strengthening the mental health education and psychological quality training of college students, help them cultivate positive and effective coping styles, and establish good interpersonal relationships to reduce interpersonal problems and alleviate loneliness. In the future, we can add longitudinal research data to further verify the relationship between college students' interpersonal relationship problems and pet attachment.

\section{REFERENCES}

[1] Dahe.cn- Henan Business Daily. Investigation: 12\% of College Students in 4 Colleges Keep Pets, and Most of Them are just Follow Su it to Try [EB/OL].

http://www.hnr.cn/news/snxw/201401/t20140103_776984.html. 2014-01-03, 06:53.

[2] Huang Ting. College Students Ignore the School Rules and Regulations to Keep Pets, and More Pets Being Abandoned after Their Graduation. $\begin{array}{lll}\text { [EB/OL]. } & \text { http://news. } & 163 . c o m\end{array}$ /17/1114/20/D37U3QPN000187VE.html.2017-11-14 20:47:00.

[3] Johnson TP, Garrity TF, Stallones L. Psychometric Evaluation of the Lexington Attachment to Pets Scale (Laps) [J]. Anthrozoos A Multidisciplinary Journal of the Interactions of People \& Animals, 1992, 5(3):160-175.

[4] Fu Na, Zheng Richang. Social Support Mechanism of Pet Dogs [J]. Chinese Mental Health Journal.2009, 23(12): 903-909.

[5] Rosenkoetter M. Health Promotion:The Influence lf Pets on Life Patterns in the Home [J]. Holistic Nursing Practice, 1991, 5:42 -51. 
[6] Sable P. Pets, Attachment and Well-being across the Life Cycle [J]. Soc Work, 2003, 40: 334-341.

[7] Stanley IH, Conwell Y, Bowen C, et al. Pet Ownership may Attenuate Loneliness among Older Adult Primary Care Patients Who Live Alone [J]. Aging Ment Health. 2014,18: 394-399.

[8] Huang Qin. Social Theory Analysis of the Phenomenon of Adolescent with Pets Companion [J]. Contemporary Youth Research. 2009, 27(10): 10-13.

[9] Harmony Rhoades, Hailey Winetrobe, Eric Rice. Pet Ownership among Homeless Youth: Associations with Mental Health, Service Utilization and Housing Status [J]. Child Psychiatry Hum Dev, 2015, 46(2): 237-244.

[10] Jitka Pikhartova, Ann Bowling, Christina Victor. Does Owning a Pet Protect Older People Against Loneliness? [J] BMC Geriatr, 2014, 14: 106-133.

[11] Fu Na, Zheng Richang. Effect of Pets on the Physical and Mental Health of Empty Nest Parents [J]. Chinese Mental Health Journal. 2003, 17(8): 569-573.

[12] Megan K. Mueller, Nancy R. Gee, Regina M. Bures. Human-animal Interaction as a Social Determinant of Health: Descriptive Findings from the Health and Retirement Study [J]. BMC Public Health, 2018, 18: 305-321.

[13] Cacioppo JT, Patrick W. Loneliness: Human Nature and the Need for Social Connection [J]. Library Journal, 2008, 19(3): 71-89.

[14] Burholt V, Scharf T. Poor Health and Loneliness in Later Life: The Role of Depressive Symptoms, Social Resources, and Rural Environments [J]. J Gerontol Series B: PsycholSci Soc Sci, 2013, 69:311-324.

[15] Li Huanhuan, Luo Xiaojun, Wang Xiang. The Relationship between College Students' Loneliness and Suicidal Tendency: Evidence from Implicit and Explicit Measures [J]. Chinese Journal of Clinical Psychology.2012, 20(6): 805- 808.

[16] Jiao Kaishan. Loneliness and the Use of Mobile Internet --Taking College Students' Use of WeChat as an Example [J]. Youth Studies. 2016, 39(4): 19-23.

[17] Stanley I. H., Conwell Y., Bowen C., et al. Pet Ownership may Attenuate Loneliness among Older Adult Primary Care Patients Who Live Alone [J]. Aging and Mental Health. 2014, 18(3): 394-399.

[18] Li Li, Mei Songli, Niu Zhimin et al. The Relationship between College Students' Loneliness and Sleep Quality: Mediating Role of Smartphone Addiction and Gender Regulation [J]. Chinese Journal of Clinical Psychology. 2016, 24(2): 345- 349.

[19] Pinquart M, SÖrensen S. Risk Factors for Loneliness in Adulthood and Old Age- a Meta- Analysis [J]. Advances in Psychology Research, 2003, 19(15): 111-143.

[20] Zhang Haixia. The Relationship among College Students' Stress Life Events, Loneliness and Suicidal Idea [J]. Occupation and Health. 2016, 32(14):1976-1979.
[21] Xie Hua, Peng Mingfang, Zhao Xue. The Status Quo of College Students' Loneliness and Its Relationship with Love and Sexua Behavior [J]. Journal of Ankang Teachers College. 2015, 27(5): 107-110.

[22] Gao Caiyun, Meng Xiangyan, Tian Xiaoguang. On the Status and Countermeasures of College Students' Loneliness [J]. China Adult Education, 2011, 20(10): 72-74.

[23] Ellemers N, Spears R, Doosje B. Sticking together or Falling apart: in-group Identification as a Psychological Determinant of Group Commitment versus Individual Mobility [J]. Journal of Personality and Social Psychology, 1997, 72(3): 617-626.

[24] Li Zhanyong. The Influence of College Students' Social Physique Anxiety on Interpersonal Relationship [J]. Chinese Journal of School Health. 2016, 37(6): 935 - 938.

[25] Murphy pm, Kupshik GA. Loneliness, Stress and Well-being: a Helper's guide [M]. New York: Tavistock Routedge. 1992.

[26] Zhang Yan, Zhou Yangen, Pei Tao. The Mediating Effect of College Students' Loneliness in Interpersonal Adaptability and Mobile Internet Dependence [J]. Chinese Mental Health Journal. 2015, 29(10): 774- 778.

[27] Compas Connor-Smith Saltzman., Compas, B. E., Connor-Smith, J. K., et al. Coping with Stress during Children and Adolescence: Problems, Progress, and Potential in Theory and Research [J]. Psychological Bulletin, 2001,127: 87-127.

[28] Wu Xiaomin. A Study on the Relationship between Pet Attachment, Pet Sadness and Coping Style of Junior Middle School Students [D]. Shanghai Normal University, 2013, 05

[29] Lei Xi, Wang Jingqun, Zhang Yuan et al. The Impact of Core Self-Evaluation on College Students' Depression: The Chain Mediation of Coping Styles and Interpersonal Relationships [J]. Chinese Journal of Clinical Psychology.2018, 26(4): 808-811.

[30] Li Xiaoling, Tang Haibo, Ming Qingsen et al. The Relationship between College Students' Loneliness and Self-Harmony: The Mediating Role of Coping Styles [J]. Chinese Journal of Clinical Psychology. 2014, 22(3): 530-532.

[31] Wood L., Martin K., Christian H., et al. The pet factor-Companion Animals as a Conduit for Getting to Know People, Friendship Formation and Social Support [J]. PLoS ONE. 2015, 10(4): 28-41.

[32] Wu Xiaomin. A Research on the Relationship between Pet Attachment, Pet Bereavement and Coping Style among Chinese Junior Schoo Student [D]. Shanghai Normal University.2013, 05.

[33] Wang Xiangdong, Wang Xilin, Ma Hong. Rating Scales for Mental Health [M]. Beijing: Chinese Mental Health Journal, 1993: 284-287.

[34] Xie Yaning. On the Reliability and Validity of the Simple Coping Style Scale [J]. Chinese Journal of Clinical Psychology. 1998, 6(2): 53-54.

[35] Zheng Richang. Psychological Diagnosis of College Students [M]. Jinan: Shandong Education Press, 1999: 324-339. 